\title{
Influence of live coral cover on coral-reef fish communities
}

\author{
J. D. Bell 1, 2 and R. Galzin ${ }^{3,4}$ \\ 1 New South Wales State Fisheries, P. O. Box N211 Grosvenor St., Sydney, N.S.W., 2000, Australia \\ 2 Present address: School of Biological Sciences, Macquarie University, North Ryde, N.S.W., 2113, Australia \\ ${ }^{3}$ Centre de l'Environnement de Mooréa, Muséum National Histoire Naturelle et École Pratique des Hautes Etudes en \\ Polynésie Française, B.P. 12 Mooréa, Polynésie Française \\ ${ }^{4}$ Present address: École Pratique des Hautes Études, Laboratoire de Biologie Marine et Malacologie, 55 rue de Buffon, 75005 \\ Paris, France
}

\begin{abstract}
The effect of percentage live coral cover on the number of fish species and individuals was determined by censusing fish from a series of reefs of comparable structural complexity, but with different proportions of live coral, in the lagoon of Mataiva Atoll, Tuamotu Archipelago. Regression analysis showed that there was a highly significant $(p<0.001)$ positive relationship between live coral cover and total number of species, number of species $250 \mathrm{~m}^{-2}$, and number of individuals $250 \mathrm{~m}^{-2}$. Changes in live coral cover estimated to be as small as $0 \%$ to $<2 \%$, and $<2 \%$ to 2 to $<5 \%$, produced significant increases in the total number of species and number of individuals $250 \mathrm{~m}^{-2}$. A change of $0 \%$ to 2 to $<5 \%$ caused a significant increase in the number of species $250 \mathrm{~m}^{-2}$. Species richness of the Chaetodontidae, Pomacentridae, Labridae, Scaridae, Acanthuridae and Gobiidae rose with increasing live coral cover, while that of Apogonidae remained relatively constant. Many of the 115 fish species recorded discriminated between sites of differing live coral cover; $68 \%$ were found only at sites with some live coral, whereas $29 \%$ were common to sites with or without live coral, and $3 \%$ were only present at sites where all coral was dead. The species compositions of sites from the same zone of live coral cover were more similar to one another than to those at sites from different zones.
\end{abstract}

\section{INTRODUCTION}

Relationships between topographic complexity of coral reefs and diversity of their fish communities (Risk, 1972; Talbot and Goldman, 1972; Luckhurst and Luckhurst, 1978; Gladfelter et al., 1980) indicate that the structure of coral-reef fish communities can be influenced by the physical complexity of the substrate. It appears that increased surface area provides a greater diversity of shelter and/or feeding sites, thus enhancing species richness.

Although some fishes depend directly on live coral for food (Hiatt and Strasburg, 1960; Randall, 1967; Hobson, 1974; Reese, 1977) investigations on how the biological nature of the substrate determines community structure are limited. Risk (1972) and Luckhurst and Luckhurst (1978) examined relationships between fish community parameters and the biological diversity of the substrate, and coral species richness, respectively. They found no significant correlations. Sale and Dybdahl $(1975,1978)$ did find differences in species associ- ated with live coral and with coralline limestone habitats bearing a few small coral colonies but concluded that differences in the 2 communities were minor. However, variations in the physical complexity of their habitats prevents assessment of the influence of living coral on fish community structure from their data. Luckhurst and Luckhurst (1978) seem to be the only workers to have examined the influence of percentage live coral cover on the structure of fish communities. They found no significant correlation between live coral cover and the number of resident and cryptic fish species associated with $9 \mathrm{~m}^{2}$ quadrats. However, their data were derived from areas with vertical rugosity values (a measure of topographic complexity) ranging from 1.1 to 4.6 at varying depths (10 to $40 \mathrm{~m}$ ).

There appear to be no studies which examine the effect of percentage live coral cover, in isolation from the significant effect of changes in habitat complexity, on the structure of entire fish assemblages from relatively large areas of reef. This is not surprising consid- 
ering that it is difficult to separate these effects. Dead coral skeletons rapidly lose their structure due to erosion whereas live coral remains structurally complex. Conditions which permit such investigation in situ are therefore rare and hard to create experimentally and maintain on an adequate scale.

From data we collected during a fish survey at Mataiva Atoll (Delesalle et al., in prep.) we describe a relatively large-scale natural situation where the effects of differential live coral cover (our estimates ranged from 0 to $10 \%$ ) on fish community structure were investigated in isolation from those of spatial heterogeneity. We found significant differences in fish species richness and density of individuals with changing live coral cover on topographically similar reefs and suggest that the presence (and amount) of live coral cover may be more important in structuring fish communities than previously thought.

\section{MATERIALS AND METHODS}

\section{Environment}

This study was carried out in the lagoon of Mataiva Atoll $\left(14^{\circ} 49^{\prime} \mathrm{S}, 148^{\circ} 34^{\prime} \mathrm{W}\right)$, the western-most member of the Tuamotu Archipelago (French Polynesia) (Fig. 1). Mataiva is a 'closed' atoll approximately $10 \mathrm{~km}$ by $5 \mathrm{~km}$ with a wide (ca. $1 \mathrm{~km}$ ) land mass along almost its entire circumference. A single pass and several 'hoa' (reef flat spillways) permit continuous water exchange with the ocean. The lagoon is bisected by submerged reefs with the same structure. This has resulted in the formation of numerous basins (average depth $=8 \mathrm{~m}$ ) within the lagoon, separated by a network of reefs (Fig. 2). All reefs were moderately complex, consisting mainly of Acropora, Pocillopora and Porites; and had a sloping profile between depths of 0.5 and $3 \mathrm{~m}$.

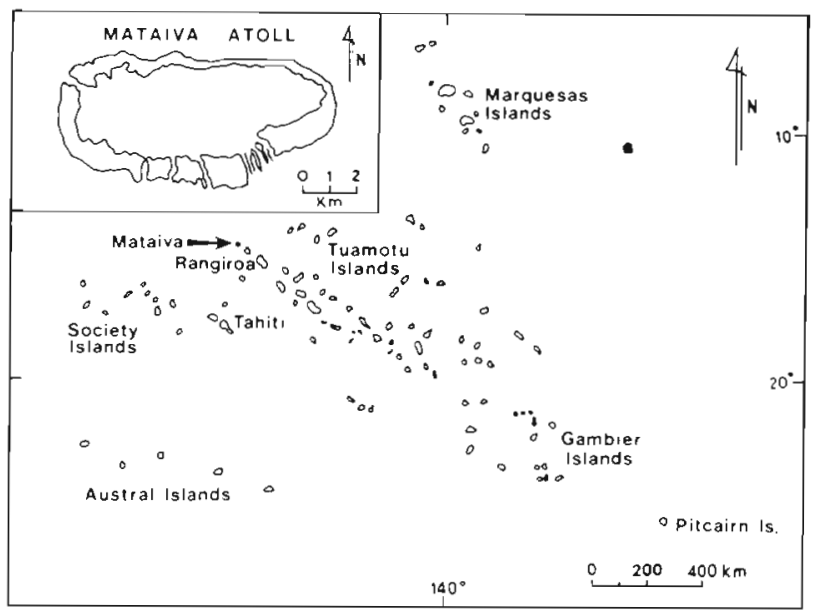

Fig. 1. Location of Mataiva Atoll

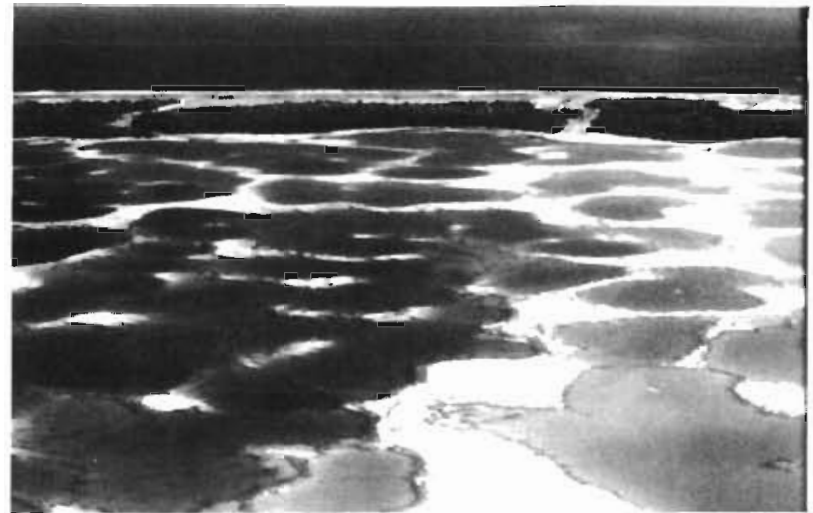

Fig. 2. Aerial photo of a portion of the lagoon at Mataiva Atoll showing the uniform nature of reefs dividing the lagoon into a series of basins

At the time of this study (9 to 20 March 1981) entire reefs surrounding several basins were completely dead while the remainder had differential quantities of live coral. Several 'zones' of live coral cover were obvious within the lagoon. Due to extremely protected conditions within the lagoon, dead and partially dead reefs were not degraded by wave action and remained as structurally complex as those with maximum coral cover. Causes of the coral mortality remain uncertain but, according to inhabitants, appear related to periods of prolonged low tide during November the previous year. We were unable to find out whether the agent causing coral mortality also killed fish.

\section{Collection of data}

Percentage live coral cover throughout the lagoon was visually assessed along $50 \mathrm{~m}$ of reef around the perimeter of a basin at points on a $1 \mathrm{~km}$ grid. Cover was estimated using the following scale; $0 \%$ (dead), $<2 \%, 2$ to $<5 \%, 5$ to $10 \%$ and $>10 \%$. We chose this method of measuring coral cover in preference to more quantitative techniques due to the limited time available to us and because data from abundance categories have proved reliable in quantifying changes in other assemblages (e.g. Frontier and Ibanez, 1974; Watling et al., 1978; Gladfelter et al., 1980). Quantitative data (percentage live cover in 7 replicate $0.5 \mathrm{~m}^{2}$ quadrats) collected by B. Salvat (pers. comm.) show that our estimates of live coral cover were reasonably accurate. He found that mean live coral cover to a depth of $4 \mathrm{~m}$ was $10.7 \%$ (with a standard deviation of 9.0 ) in our $>10 \%$ zone.

We selected sampling sites consisting of a section of reef surrounding a basin within each zone of coral cover. All sites had a depth range of 0 to $3 \mathrm{~m}$ and, as mentioned previously, were of similar structural com- 


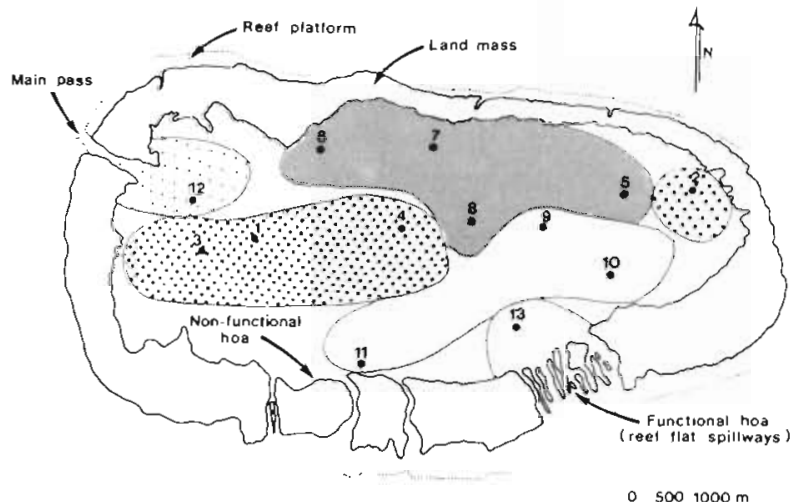

$[\because 30 \% \square<2 \% \quad \square 2-<5 \% \square-\square^{5} \cdot 10 \% \square>10 \% \quad \underbrace{5001000 \mathrm{~m}}$

Fig. 3. Zones of percentage live coral cover and location of the ocean pass, functional hoa and fish sampling sites in the lagoon at Mataiva Atoll

plexity. The large scale of our sampling sites meant that we were unable to employ the chain-link method of measuring structural complexity used by Risk (1972) and Luckhurst and Luckhurst (1978) for small areas (up to $9 \mathrm{~m}^{2}$ )

The distribution of live coral cover zones and location of sampling sites within the lagoon are shown in Fig. 3. The limited areas of 5 to $10 \%$ and $>10 \%$ live coral cover prevented an orthogonal sampling design. Four sites were established in $0 \%$ and $<2 \%$ zones, 3 sites in the 2 to $<5 \%$ zone and 1 site in each of the 5 to $10 \%$ and $>10 \%$ zones. Fish communities associated with each site were assessed in 2 ways. We censused the abundances of conspicuous species within a $250 \mathrm{~m}^{2}$ transect area by placing a $50 \mathrm{~m}$ line along the reef at a depth of $1.5 \mathrm{~m}$ and recording the numbers of each species $2.5 \mathrm{~m}$ to either side. Data were collected once by each of us at a 5 min interval. There was consistency between our counts (data were significantly correlated at the $0.1 \%$ level, using Pearsons correlation coefficient, for 10 of the 13 pairs of samples) and so we averaged our abundance estimates. A total species list for each site was then compiled by supplementing the transect data with species collected by poisoning a Porites coral head (1.5 to $2.0 \mathrm{~m}$ in diameter) with $0.5 \mathrm{~kg}$ of rotenone powder and those seen by us along $100 \mathrm{~m}$ of reef (to a depth of $3 \mathrm{~m}$ ) during a $20 \mathrm{~min}$ period.

\section{Data analysis}

Linear regression was used to investigate the effects of percentage live coral cover (mid-point values were used for the $<2 \%, 2$ to $<5 \%$ and 5 to $10 \%$ categories) on the numbers of species and individuals $250 \mathrm{~m}^{-2}$ and the total number of species recorded at each site. Scheffe's multiple comparisons test was used to test whether small increases in percentage live coral cover over the lower end of the range (e.g. from $0 \%$ to $<2 \%$ and $<2 \%$ to 2 to $<5 \%$ ) caused significant changes in the means of these parameters.

Grovhoug and Henderson (1978) found that the number of fish species and individuals in the lagoon at Canton Atoll decreased with distance away from the pass into the lagoon, and Gladfelter et al. (1980) suggested that fish species diversity on patch reefs close to the barrier reef at Enewetak Atoll was higher than those further away because they received more immigrants from the larger reef. As many of our sites had been subjected to conditions deletereous to coral (and perhaps fish) and differ in distance from the nearest functional pass or hoa, presumably major sources of larval recruits and immigrants, we used multiple regression to separate the effects of distance and percentage live coral cover on the three parameters of community structure.

Similarities between sites were determined by classifying presence/absence data with the agglomerative program MULBET (Williams, 1976), which uses Jaccard's coefficient. The data were then ordinated using the principal co-ordinates analysis program GOWER (Williams, 1976).

\section{RESULTS}

\section{The fish fauna}

We recorded 115 species of fish at the 13 sites. Sixtyone species were observed in $250 \mathrm{~m}^{2}$ transects and a further 37 and 17 species were added by observations over a $100 \mathrm{~m}$ section of reef and through poison stations, respectively. Species associated with each site and the abundances of those observed in each $250 \mathrm{~m}^{2}$ transect are given in Table 1.

\section{Discrimination among coral zones by fish}

There was considerable evidence that several species avoided sites without live coral. Seventy-eight species (68\% of the total number of species recorded) were present at sites with some live coral but absent from those with none (Table 1). The amount of live coral also influenced the distribution of many species, e.g. 29 of the above 78 species were associated only with the 2 sites of highest coral cover. By contrast, only 4 species $(3 \%)$ were recorded exclusively from sites without live coral. Three of these species were recorded once as single individuals. Thirty-three species $(29 \%)$ were recorded at sites with and without live coral. Fourteen of these were common to all sites 
Table 1. Fish species recorded at sites with differing live coral cover in Mataiva Lagoon; numbers in brackets; mean abundances $250 \mathrm{~m}^{-2}$

\begin{tabular}{|c|c|c|c|c|c|c|c|c|c|c|c|c|c|}
\hline \multirow{3}{*}{$\begin{array}{l}\text { Family } \\
\text { Species }\end{array}$} & \multicolumn{13}{|c|}{ Zones of percentage live coral cover (and sites within zones) } \\
\hline & \multicolumn{4}{|c|}{0} & \multicolumn{4}{|c|}{$<2$} & \multicolumn{3}{|c|}{$2-<5$} & \multirow{2}{*}{$\begin{array}{c}5-10 \\
12\end{array}$} & $>10$ \\
\hline & 1 & 2 & 3 & 4 & 5 & 6 & 7 & 8 & 9 & 10 & 11 & & 13 \\
\hline MYLIOBATIDAE & & & & & & & & & & & & & \\
\hline Aetobatis narinari & $x$ & & & $\times$ & & & & $x$ & $x$ & & $x$ & & \\
\hline SYNODONTIDAE & & & & & & & & & & & & & \\
\hline Saurida gracilis & $x$ & & & & & & & & & & & & \\
\hline $\begin{array}{l}\text { MURAENIDAE } \\
\text { Echidna nebulosa }\end{array}$ & & $x$ & & & & & & & & & & & \\
\hline $\begin{array}{l}\text { Echidna nebulosa } \\
\text { Gymnothorax javanicus }\end{array}$ & & & & & & $x$ & & & $x$ & & & $\times(1)$ & \\
\hline Gymnothorax margaritophorus & & & & & & & & & $x$ & & $x$ & & \\
\hline Gymnothorax sp. & & & & & & & & & & & & $x$ & $x$ \\
\hline BELONIDAE & & & & & & & & & & & & & \\
\hline Tylosurus crocodilus & & & & & $x$ & & & & & & & & \\
\hline HEMIRHAMPHIDAE & & & & & & & & & & & & & \\
\hline Hyporhamphus acutus & & & & $x$ & & & & & & $x$ & & & \\
\hline HOLOCENTRIDAE & & & & & & $x$ & & & & $x$ & $x$ & $x$ & ૫ \\
\hline $\begin{array}{l}\text { Adiaryx spinifer } \\
\text { Flammeo opercularis }\end{array}$ & & & & & & & $x$ & & $x$ & $x$ & $\hat{\lambda}$ & $\widehat{\lambda}$ & $\hat{\lambda}$ \\
\hline Flammeo sammara & & $x$ & & & & & & $\times(1)$ & $x$ & & $x$ & & \\
\hline Myripristis murdjan & $x$ & & & & & & & & & & $x$ & $x$ & $x$ \\
\hline Mypristis sp. & $x$ & & $x$ & & & & & & & & & & \\
\hline FISTULARIDAE & & & & & & & & & & & & & \\
\hline Fistularia commersonii & & & & & & & & & & & & & $x$ \\
\hline SPHYRAENIDAE & & & & & & & & & & & & & \\
\hline Sphyraena barracuda & & & & & & & & & & & & & $\times$ \\
\hline SCORPAENIDAE & & & & & & & & & & & & & \\
\hline $\begin{array}{l}\text { Scorpaenodes guamensis } \\
\text { SERRANIDAE }\end{array}$ & & & & & & & & & & & & $x$ & \\
\hline $\begin{array}{l}\text { SERRANIDAE } \\
\text { Cephalopholis argus }\end{array}$ & & & & & & & & & & & & $\times(2)$ & $x$ \\
\hline Epinephelus merra & & & & & & & & & $x$ & & & $x$ & \\
\hline Epinephelus microdon & & $\times(1)$ & $x$ & & $\times(1)$ & & $x$ & & & & & $x$ & $x$ \\
\hline GRAMMISTIDAE & & & & & & & & & & & & & \\
\hline Grammistes sexlineatus & & & & $x$ & $x$ & & & & & & & & \\
\hline Pseudogramma polyacantha & & & & & & & & & & & & & $x$ \\
\hline APOGONIDAE & & & & & & & & & & & & & \\
\hline Apogon exostigma & & & & & & $\times$ & $\times(1)$ & & & $x$ & $x$ & $x$ & \\
\hline $\begin{array}{l}\text { Apogon marmoratus } \\
\text { Apogon novemfasciatus }\end{array}$ & & & $x$ & $x$ & $x$ & $\times$ & $\times$ & $\times$ & $\begin{array}{l}x \\
x\end{array}$ & $x$ & $\lambda$ & $\hat{x}(1)$ & \\
\hline Apogon savayensis & $x$ & $x$ & $x$ & $x$ & & & & & & $\times$ & $x$ & & \\
\hline Cheilodipterus macrodon & & & & & & & & & & $x$ & & & \\
\hline Cheilodipterus quinquelineatus & $\times(1)$ & $\times(2)$ & $\times(1)$ & $x$ & $\times(1)$ & $\times(1)$ & $\times(2)$ & $\times(2)$ & $\times(4)$ & $\times(1)$ & $\times(5)$ & $\times(3)$ & $x$ \\
\hline Fowleria sp. & & & & & & & & & & & & & $x$ \\
\hline Pseudamia sp. & & & $x$ & & & $\times$ & & & & & & & \\
\hline CARANGIDAE & & & & & & & & & & & & & \\
\hline Caranx melampygus & & & & & $x$ & & & & $\times(1)$ & $x$ & $x$ & & $x$ \\
\hline LUTJANIDAE & & & & & & & & & & & & & \\
\hline Lutjanus fulvus & $x$ & $x$ & $\times(1)$ & $\times(2)$ & $\times(3)$ & $\times(12)$ & $x$ & $x$ & $\times(1)$ & $\times(4)$ & $x$ & $\times(1)$ & \\
\hline Lutjanus gibbus & & & & & & & & & & & & $x$ & $x$ \\
\hline MULLIDAE & & & & & & & & & & & & & \\
\hline Mulloidichthys flavolineatus & & $\times(1)$ & $x$ & $\times(1)$ & $\times(6)$ & $x$ & $\times(4)$ & $x$ & $x$ & $x$ & & & \\
\hline Parupeneus barberinus & & & $x$ & $\times$ & & & & & & & & & \\
\hline Parupeneus bifasciatus & & & & & & & & & & & & & $\times(1)$ \\
\hline Parupeneus trifasciatus & & & & & & & $x$ & & $x$ & $\times(3)$ & $x$ & $x$ & $\times(5)$ \\
\hline LETHRINIDAE & & & & & & & & & & & & & \\
\hline Monotaxis grandoculis & & & & & & & & & & & & & $x$ \\
\hline CHAETODONTIDAE & & & & & & & & & & & & & \\
\hline Chaetodon auriga & & $\times(1)$ & $x$ & $x$ & $\times(5)$ & $x$ & $x$ & $x$ & $\times(1)$ & $x$ & $\times(1)$ & $x(1)$ & $\times(6)$ \\
\hline Chaetodon bennetti & & & & & $x$ & & & $x$ & $\times(2)$ & $x$ & & & \\
\hline Chaetodon citrinellus & & & & & & & & & & & & & $\times(3)$ \\
\hline Chaetodon ephippium & & $\times(1)$ & $\times(1)$ & $x$ & $\times(2)$ & $\times(1)$ & $\times$ & $\times(1)$ & $\times(2)$ & $\times(9)$ & $\times(2)$ & $\times(4)$ & $x(2)$ \\
\hline Chaetodon lineolatus & & & & & & & & & & & & & $x$ \\
\hline Chaetodon lunula & & & & & $\times(1)$ & $\times(1)$ & $\times(1)$ & $\times(1)$ & $\times(2)$ & $x$ & & $x$ & $\times(10)$ \\
\hline Chaetodon quadrimaculatus & & & & & & & & $x$ & & & & & \\
\hline Chaetodon semeion & & & & & & & & & & $x$ & & & $x$ \\
\hline Chaetodon trifasciatus & & & & & $x$ & $x$ & $x$ & $\times(2)$ & $x$ & $\times(16)$ & $\times(4)$ & $x$ & $\times(8)$ \\
\hline Chaetodon ulietensis & & & & & $\times(1)$ & & & & $x$ & $\times(1)$ & $\times(1)$ & & $\times(6)$ \\
\hline Chaetodon unimaculatus & & & & & & & & & & & & $x$ & $\times(1)$ \\
\hline Chaetodon vagabundus & & & & & & & & & & $\times(1)$ & $x$ & & \\
\hline POMACANTHIDAE & & & & & & & & & & & & & \\
\hline Centropyge flavissimus & & & & & & & & & & & $\times(1)$ & $x$ & $x$ \\
\hline POMACENTRIDAE & & & & & & & & & & & & & \\
\hline Abudefduf sexfasciatus & & & $x$ & & & & & $\times(1)$ & & & & & \\
\hline $\begin{array}{l}\text { Chromis caerulea } \\
\text { Chrysiptera leucopomus }\end{array}$ & $\times(7)$ & $\times(15)$ & $x$ & $x$ & & $\times(27)$ & $\times(56)$ & $\times(27)$ & $\times(92)$ & $\times(30)$ & $\times(24)$ & $\times(69)$ & $x$ \\
\hline & & & & $x$ & & $\times(2)$ & $\times(3)$ & $\times(1)$ & $\times(9)$ & $\times(17)$ & $\times(15)$ & $\begin{array}{l}\times(1) \\
\times(40)\end{array}$ & $\times(7)$ \\
\hline Pomacentrus coelestis & & & $x$ & $x$ & $x$ & $\times(1)$ & $\times(8)$ & $\times(1)$ & $\times(11)$ & $x$ & $\times(11)$ & $x$ & $x$ \\
\hline
\end{tabular}


Table 1. Continued

\begin{tabular}{|c|c|c|c|c|c|c|c|c|c|c|c|c|c|}
\hline \multirow{3}{*}{$\begin{array}{l}\text { Family } \\
\text { Species }\end{array}$} & \multicolumn{13}{|c|}{ Zones of percentage live coral cover (and sites within zones) } \\
\hline & \multicolumn{4}{|c|}{0} & \multicolumn{4}{|c|}{$<2$} & & $-<5$ & & $5-10$ & $>10$ \\
\hline & 1 & 2 & 3 & 4 & 5 & 6 & 7 & 8 & 9 & 10 & 11 & 12 & 13 \\
\hline POMACENTRIDAE & & & & & & & & & & & & & \\
\hline Pomacentrus sp. & & & & & & & & & & $\times$ & & & \\
\hline Stegastes albifasciatus & $x$ & & & & & & & & & & & & \\
\hline $\begin{array}{l}\text { Stegastes nigricans } \\
\text { LABRIDAE }\end{array}$ & & & & & & $x$ & & & & & $\times(3)$ & $\times(1)$ & $\times(15)$ \\
\hline Bodianus sp. & & & & & & & & & & $\times(1)$ & & & $x$ \\
\hline Cheilinus chlorurus & & & & & & & & & & & $x$ & & $x$ \\
\hline Cheilinus oxycephalus & & & & & & & & & & & & & $x$ \\
\hline Cheilinus trilobatus & & & & & & & & & & & & & $\times(1)$ \\
\hline Cheilinus undulatus & & & & & & & & & & & $x$ & & \\
\hline Cheilinus sp. & $x$ & & $x$ & & $x$ & & & $x$ & & & & $\times(1)$ & $x$ \\
\hline Coris aygula & & & & & & & & & & & & & $x$ \\
\hline Cons gaimardi & & & & & $\times(1)$ & & & & & & & & \\
\hline Gomphosus varius & & & & & & & & & & & $x$ & & $\times(4)$ \\
\hline Halichoeres hortulanus & & $x$ & $x$ & & & & $x$ & $x$ & $x$ & $x$ & $\times(1)$ & $\times(20)$ & $\times(5)$ \\
\hline Halichoeres marginatus & & & & & & & & $\times(1)$ & & & & & $\times$ \\
\hline Halichoeres trimaculatus & & & & & & & & & & & $x$ & & \\
\hline Halichoeres sp. & & & & $x$ & $x$ & & & $x$ & & $x$ & & $\times(3)$ & \\
\hline Labroides dimidiatus & & & & & & & & & & & & & $\times(1)$ \\
\hline Pseudocheilinus octotaenia & & & & & & & & & & & $x$ & $\times(1)$ & $\times(1)$ \\
\hline$P_{\text {sqeudocheilinus tetrataenia }}$ & & & & & & & & & & & & $x$ & \\
\hline Stethojulis bandanensis & & & & & & & & & $\times$ & & $x$ & $x$ & $\times(1)$ \\
\hline Thalassoma amblycephalus & & & & & & & & & & & & $\times(3)$ & $\times(1)$ \\
\hline Thalassoma hardwicki & $x$ & & & $x$ & & & $x$ & & $\times(1)$ & $\times(2)$ & $\times(2)$ & $\times(3)$ & $\times\left(x_{1}\right)$ \\
\hline Thalassoma quinquevittata & & & & & & $x$ & & & $x$ & & $\times(4)$ & $x$ & $\times(2)$ \\
\hline Wetmorella sp. & & & $x$ & & & & & & & & & $\times(1)$ & $\times$ \\
\hline Unidentified labrid & & & & & & & & & & & & & $\times(1)$ \\
\hline SCARIDAE & & & & & & & & & & & & & \\
\hline Hipposcarus longiceps & & & & $x$ & $\times(2)$ & $x$ & & & $x$ & $x$ & $x$ & & $\times(3)$ \\
\hline Scarus ghobban & & & & & $\times(2)$ & $\times(1)$ & $x$ & $x$ & $\times$ & $\times(2)$ & $x$ & & $\times(2)$ \\
\hline Scarus gibbus & & & & & & & & & & & $x$ & & $\times$ \\
\hline Scarus globiceps & & & & & & & & & & & & & $x$ \\
\hline Scarus oviceps & & & & & & $x$ & & & & $\times(2)$ & & & $x$ \\
\hline Scarus sordidus & $x$ & & $x$ & $x$ & $\times(5)$ & $x$ & $\times(5)$ & $\times(2)$ & $\times(1)$ & $\times(65)$ & $\times(9)$ & $\times(7)$ & $\times(34)$ \\
\hline Scarus sp. & $\times(1)$ & $\times(1)$ & $\times(2)$ & $\times(1)$ & $\times(24)$ & $x$ & $\times(1)$ & $\times(8)$ & $\times(4)$ & $x$ & $\times(11)$ & $\times(53)$ & $\times(6)$ \\
\hline ACANTHURIDAE & & & & & & & & & & & & & \\
\hline Acanthurus nigricauda & & & & & & & & & & & & $x$ & $x$ \\
\hline Acanthurus triostegus & $x$ & $x$ & $\times(1)$ & $\times(1)$ & $\times(5)$ & $x$ & $x$ & $\times(1)$ & $\times(2)$ & $\times(14)$ & $\times(15)$ & $\times(6)$ & $\times(20)$ \\
\hline Acanthurus xanthopterus & & & & $x$ & $x$ & & & & & & & & $x$ \\
\hline Acanthurus sp. & & & & & & & & & & $x$ & & & $x$ \\
\hline Ctenochaetus striatus & & & & & & & & & $x$ & $\times(1)$ & $x$ & $x$ & $\times(11)$ \\
\hline Naso lituratus & & & & & & & & & & & & & $x$ \\
\hline Naso unicomis & & & & & & & & & & & & & $x$ \\
\hline Zebrasoma veliferum & & & & & $\times(3)$ & & & & $x$ & $\times(2)$ & $\times(1)$ & $x$ & $\times(4)$ \\
\hline GOBIIDAE & & & & & & & & & & & & & \\
\hline Amblygobius phalaena & $\times(4)$ & $\times(11)$ & $\times(14)$ & $\times(3)$ & $\times(2)$ & $\times(4)$ & $\times(9)$ & $\times(16)$ & $\times(13)$ & $\times(1)$ & $\times(35)$ & $\times(13)$ & $\times(8)$ \\
\hline Amblygobius nocturnus & $\times(4)$ & $\times(2)$ & $\times(5)$ & $\times(1)$ & $x$ & $\times$ & $\times(2)$ & $x$ & $x$ & & $\times(1)$ & $x$ & \\
\hline Asterropteryx semipunctatus & & & $x$ & & & & & $x$ & $\times(1)$ & $x$ & $x$ & $\times(7)$ & $\times(20)$ \\
\hline Callogobius sp. A & & & & & & $x$ & & & & $x$ & & & \\
\hline Callogobius sp. B & & & & & & $x$ & $x$ & & & & & & \\
\hline Eviota afelei & & & & & & $x$ & $x$ & $x$ & $x$ & $x$ & $x$ & $x$ & $x$ \\
\hline Eviota infulata & & & & & & $x$ & $x$ & & & $x$ & $x$ & & \\
\hline Fusigobius neophytus & & & & & & & & & & & & & $x$ \\
\hline Gnatholepis sp. & & & & & & & & & & & & $x$ & $x$ \\
\hline Ptereleotris microlepis & & & & & & & & & & & $x$ & & $x$ \\
\hline Vanderhorstia sp. & & & & & & & & & & & $x$ & $x$ & \\
\hline BLENNIIDAE & & & & & & & & & & & & & \\
\hline Enchelyurus ater & & & & & & $x$ & $x$ & $x$ & $x$ & $x$ & $x$ & & $x$ \\
\hline Istiblennius periophthalmus & & & & & & & & & & & & $\times(1)$ & \\
\hline CALLIONYMIDAE & & & & & & & & & & & & & \\
\hline Callionymus sp. & & & & & $x$ & & & & & & & & \\
\hline BOTHIDAE & & & & & & & & & & & & & \\
\hline Bothus mancus & & & & & & & $\times(1)$ & & & & & $x$ & \\
\hline BALISTIDAE & & & & & & & & & & & & & \\
\hline Balistoides viridescens & & & & $x$ & & & & & & & & & $x$ \\
\hline Rhinecanthus aculeatus & & & & & & & & & $x$ & & $\times(1)$ & $\times(1)$ & $\times(1)$ \\
\hline TETRAODONTIDAE & & & & & & & & & & & & & \\
\hline Canthigaster bennetti & & & & & & & & & & & & $\times(1)$ & $\times(4)$ \\
\hline Canthigaster janthinoptera & & & & & $\times(1)$ & $\times(1)$ & & & $\times(3)$ & $x$ & $\times(4)$ & $\times(1)$ & $x$ \\
\hline OSTRACIIDAE & & & & & & & & & & & & & \\
\hline Ostracion cubicus & & & & & $x$ & & & & & & $\times(1)$ & & $x$ \\
\hline Total no. of species & 16 & 15 & 23 & 24 & 30 & 31 & 28 & 29 & 40 & 42 & 49 & 52 & 73 \\
\hline No. of species $250 \mathrm{~m}^{-2}$ & 5 & 9 & 7 & 6 & 17 & 10 & 12 & 14 & 17 & 18 & 22 & 27 & 32 \\
\hline No. of individuals $250 \mathrm{~m}^{-2}$ & 17 & 35 & 25 & 9 & 65 & 51 & 93 & 65 & 150 & 172 & 152 & 246 & 201 \\
\hline
\end{tabular}



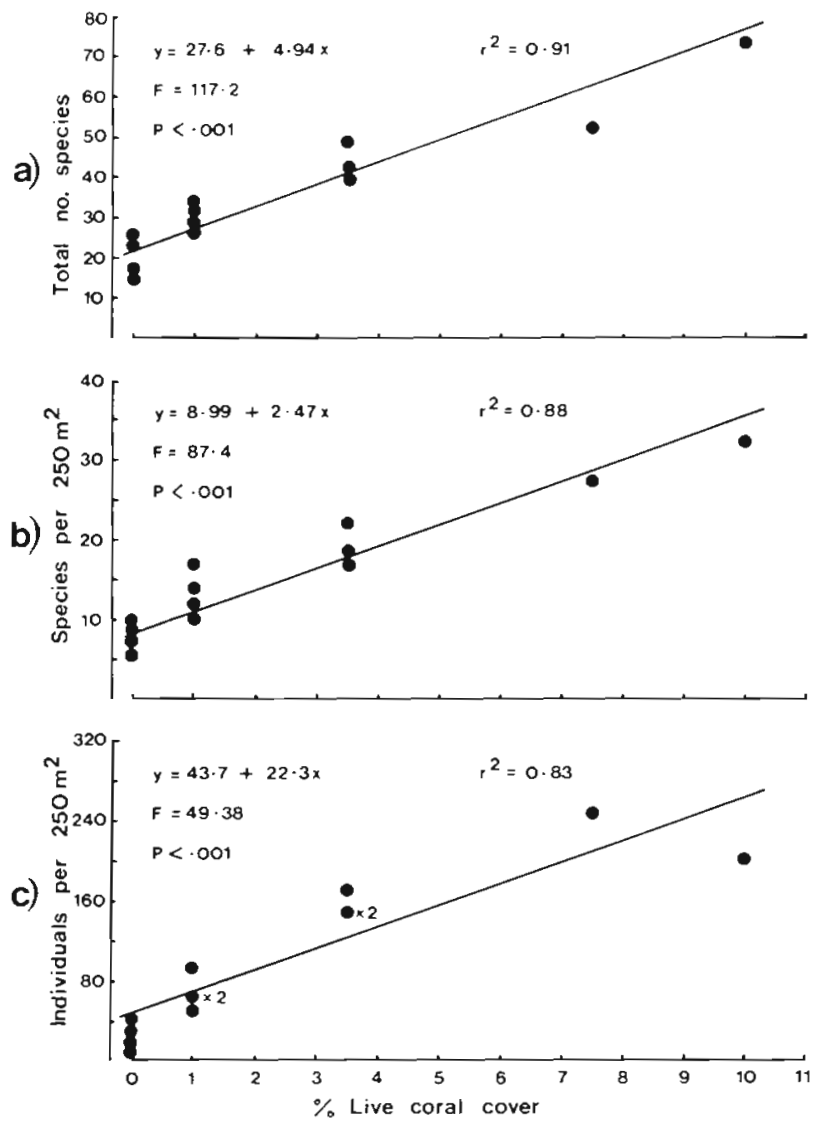

Fig. 4. Linear regressions of percentage live coral cover against (a) total number of species, (b) number of species $250 \mathrm{~m}^{-2}$ and (c) number of individuals $250 \mathrm{~m}^{-2}$

or present at most sites in each zone of live coral cover. Seven of them (Cheilodipterus quinquelineatus, Lutjanus fulvus, Chaetodon auriga, C. ephippium, Scarus sp., Amblygobius phalaena and A. nocturnus) showed little discrimination between sites on the basis of their abundance $250 \mathrm{~m}^{-2}$. However, the abundances of the remaining 7 species (Chromis caerulea, Pomacentrus coelestis, Dasyllus aruanus, Halichoeres hortulanus,

Table 2. Mean number of fish species in the major families from each zone of percentage live coral cover; values from 5 to $10 \%$ and $>10 \%$ zones are not means but represent single records

\begin{tabular}{|lcccrc|}
\hline \multirow{2}{*}{ Family } & \multicolumn{6}{c}{$\begin{array}{c}\text { Zone of percentage live coral cover } \\
\end{array}$} & 0 & $<2$ & $2-<5$ & $5-10$ & $>10$ \\
\hline Apogonidae & 2.8 & 2.5 & 3.3 & 3 & 1 \\
Chaetodontidae & 1.5 & 5.0 & 6.3 & 5 & 9 \\
Pomacentridae & 2.3 & 3.0 & 3.7 & 5 & 4 \\
Labridae & 2.3 & 2.5 & 5.7 & 10 & 17 \\
Scaridae & 2.0 & 3.8 & 4.7 & 2 & 7 \\
Acanthuridae & 1.3 & 1.3 & 3.3 & 4 & 8 \\
Gobiidae & 2.3 & 3.8 & 5.3 & 6 & 7 \\
\hline
\end{tabular}

Thalassoma hardwicki, Scarus sordidus and Acanthurus triostegus) generally increased as live coral cover increased (Table 1).

\section{Species richness}

Number of species $250 \mathrm{~m}^{-2}$ and total number of species increased with increasing live coral cover (Fig. 4). Linear regression removed 89 and $91 \%$ of the variance in these 2 estimates of diversity, respectively. The slope of each regression line was significantly different from zero $(\mathrm{p}<.001)$. The species richness of the dominant fish families in the lagoon increased as live coral cover improved (Table 2). The only exception to this was the Apogonidae which did not demonstrate a consistent trend in richness.

Scheffe's multiple comparisons test showed that increases in live coral cover as small as those from $0 \%$ to $<2 \%$ and $<2 \%$ to 2 to $<5 \%$ caused significant changes in the total number of species in the community. However, a change in live coral cover from $0 \%$ to 2 to $<5 \%$ was required to increase significantly the number of conspicuous species (Table 3 ).

Table 3. Matrix of values for Scheffe's multiple comparisons test for comparison of means in (1) total number of species, (2) number of species $250 \mathrm{~m}^{-2}$, and (3) number of individuals $250 \mathrm{~m}^{-2}$, between the 3 lowest zones of percentage live coral cover; df $(2,6)$

\begin{tabular}{|clc|}
\hline $\begin{array}{c}\text { Zone of percentage } \\
\text { live coral cover }\end{array}$ & $<2 \%$ & $2-<5 \%$ \\
\hline $0 \%$ & (1) $7.0^{\circ}$ & (1) $33.9^{\circ}$ \\
& (2) $3.4^{\circ}$ & (2) $13.5^{\circ}$ \\
& (3) $6.4^{\circ}$ & (3) $57.4^{\circ}$ \\
$<2 \%$ & & (1) $10.1^{\circ}$ \\
& & (2) $3.4^{\circ}$ \\
$=\mathrm{p}<.05$ & (3) $25.6^{\circ}$ \\
\hline
\end{tabular}

Multiple regression analysis showed that even when distance (from the nearest pass or functional hoa) was used first in the regression, percent live coral cover accounted for significant further variance in both the number of species $250 \mathrm{~m}^{-2}$ and the total number of species (Table 4). On the other hand, when live coral was used first, it removed virtually all the variation which was also correlated with distance. Thus even if distance is presumed to have a significant biological effect, live coral cover is still also important. However, the converse is not true; if live coral is supposed to be the primary biological effect, distance need not be invoked to explain further variance. 
Table 4. Additional percent variance explained by coral cover $\left(x_{1}\right)$ and distance $\left(x_{2}\right)$ when used second in multiple regression. Significance levels provided for each variable when regressed alone are derived from linear regression

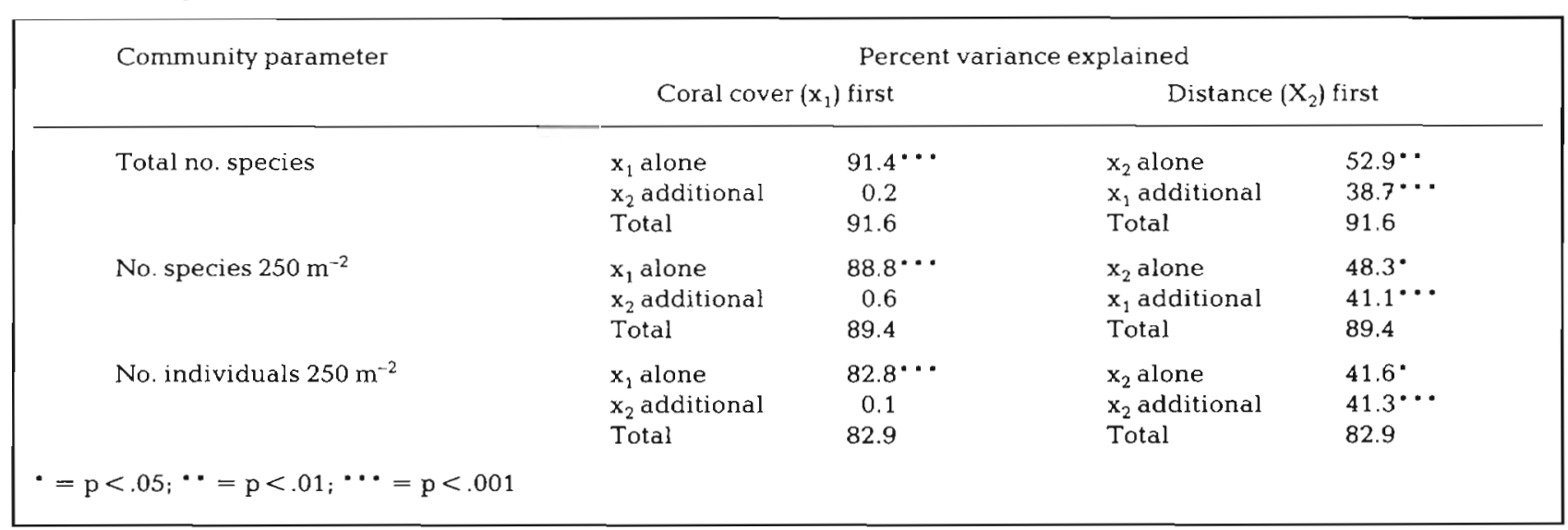

\section{Number of individuals}

The number of individuals $250 \mathrm{~m}^{-2}$ increased with percentage live coral cover $\left(r^{2}=0.83, p<.001\right)$ (Fig. 4) and was significantly correlated with the number of species $250 \mathrm{~m}^{-2}$ (Pearson's $\mathrm{r}=0.92, \mathrm{p}<.001$ ). Small changes in live coral cover (e.g. from $0 \%$ to $<2 \%$ ) significantly increased the number of individuals $250 \mathrm{~m}^{-2}$ (Table 3). Multiple regression showed that the effects of distance and live coral cover on the number of individuals $250 \mathrm{~m}^{-2}$ were the same as those described for species richness (Table 4).

\section{Faunal similarity of sites}

The ordination (Fig. 5) extracted $44 \%$ of the variance on the first two vectors. Samples from the same zone of live coral grouped together and were clearly separated from those collected in other zones. Vector 1 scores were significantly correlated with percentage live coral cover data (Spearman rank correlation coefficient $=0.97, \mathrm{p}<.001$ ) and the 20 species most significantly correlated with Vector 1 were associated only with sites of live coral cover (Table 5). Vector 1 is therefore presumed to represent percentage live coral cover.

\section{DISCUSSION}

Our data show that percentage live coral cover should be added to the variety of factors capable of determining the structure of coral reef fish communities. In isolation from the effects of reef size, water depth and structural complexity, small changes in the amount of live coral cover produced significant changes in species richness and abundance of fishes.
We do not know whether individual species were attracted to areas with more live coral, thus increasing fish abundance at those sites or whether sites with more coral favoured fish in general, with the logical consequence that more species were found there. However, similarities between samples (Fig. 5), which show that many of the species added to sites within each of the zones of higher live coral cover were the same, suggest that several species have specific live coral cover requirements and do not join the community until sufficient live coral is available (e.g. $68 \%$ of species were found only where there was some live coral). This type of relationship would be predicted by most reef ecologists for some common groups of reef fish, e.g. the Chaetodontidae (many of which are obli-

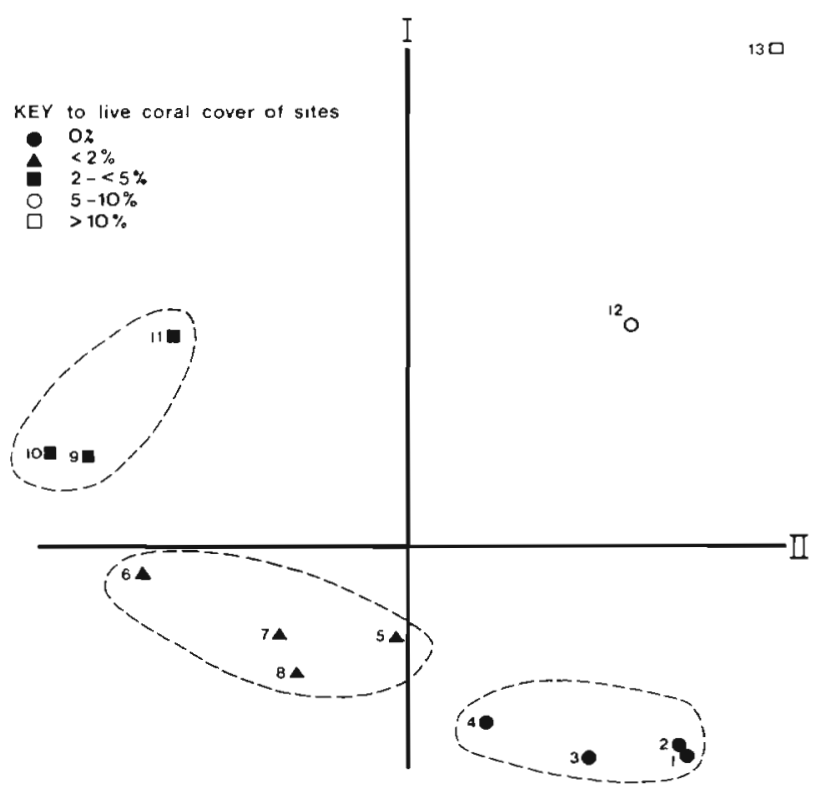

Fig. 5. Principal co-ordinates analysis of species occurrence data for each sample plotted against Vectors I and II 
Table 5. The 20 fish species most significantly correlated with Vector I in the ordination, and zones of percentage live coral cover from which they were recorded

\begin{tabular}{|c|c|c|c|c|c|c|}
\hline \multirow[t]{2}{*}{ Species } & \multirow[t]{2}{*}{$\mathrm{r}$} & \multicolumn{5}{|c|}{ Zone of percentage live coral cover } \\
\hline & & 0 & $<2$ & $2-<5$ & $5-10$ & $>10$ \\
\hline Ctenochaetus striatus & $0.858^{\cdots}$ & & & $x$ & $\times$ & $x$ \\
\hline Rhinecanthus aculeatus & $0.829^{\cdots} \cdots$ & & & $x$ & $\times$ & $x$ \\
\hline Centropyge flavissimus & $0.828^{\cdots} \cdot$ & & & $x$ & $x$ & $x$ \\
\hline Gomphosus varius & $0.828^{\cdots} \cdots$ & & & $\times$ & & $\times$ \\
\hline Stethojulis bandanensis & $0.828 \cdots$ & & & $x$ & $\times$ & $x$ \\
\hline Adioryx spinifer & $0.817^{\cdots} \cdot$ & & $x$ & $\times$ & $x$ & $\times$ \\
\hline Thalassoma quinquevittata & $0.817^{\cdots}$ & & $x$ & $x$ & $x$ & $x$ \\
\hline Parupeneus trifasciatus & $0.771 \cdots$ & & $x$ & $x$ & $x$ & $x$ \\
\hline Zebrasoma veliferum & $0.769^{\cdots}$ & & $x$ & $x$ & $\times$ & $\times$ \\
\hline Cephalopholis argus & $0.749^{\cdots}$ & & & $x$ & $x$ & \\
\hline Lutjanus gibbus & $0.749^{*}$ & & & & $x$ & $\times$ \\
\hline Chaetodon unimaculatus & $0.749^{\circ} \cdot$ & & & & $\times$ & $x$ \\
\hline Thalassoma amblycephalus & $0.749^{\cdots}$ & & & & $x$ & $x$ \\
\hline Acanthurus nigricauda & $0.749^{\cdots}$ & & & & $x$ & $x$ \\
\hline Cnatholepis sp. & $0.749^{\cdots}$ & & & & $x$ & $x$ \\
\hline Canthigaster bennetti & $0.749^{*}$ & & & & $x$ & $x$ \\
\hline Canthigaster janthinoptera & $0.748^{\cdots}$ & & $x$ & $x$ & $x$ & $x$ \\
\hline Cheilinus chlorurus & $0.741^{\cdots}$ & & & $x$ & & $x$ \\
\hline Ptereleotris microlepis & $0.741^{\cdots}$ & & & $x$ & & $x$ \\
\hline Scarus gibbus & $0.741 \cdots$ & & & $x$ & & $x$ \\
\hline
\end{tabular}

gate coral feeders, Hobson, 1974; Reese, 19; 7). A similar trend in the occurrence and abundance of the Labridae, which feed on vagile invertebrates (Hiatt and Strasburg, 1960; Vivien, 1973; Hobson, 1974), is harder to explain. Perhaps these fishes depend on prey whose occurrence and abundance are related to the availability of living coral. Such relationships exist among coral reef invertebrates; for example, Coles (1980) found that the number of symbiont decapod species was significantly correlated with live coral cover whereas nonsymbiont species increased as live coral cover declined.

If the amount of live coral dictates the nature of prey, then fishes at sites both with and without live coral should be generalist feeders on reef associated organisms or obtain their food elsewhere. This was generally true in Mataiva lagoon. Of the 14 species present at sites in all zones of live coral cover the Pomacentridae are planktivores and Lutjanus fulvus feeds on the benthos of soft substrates (Randall, 1955). The gobies Amblygobius phalaena and $A$. nocturnus live, and presumably feed, on the soft substrate around the fringe of reefs. Among the members of this group which feed on reef associated organisms the apogonid Cheilodipterus quinquelineatus is a generalist predator of larger crustaceans and small fish (Hiatt and Strasburg, 1960) and Chaetodon auriga and C. ephippium have some of the broadest diets in their family (Hiatt and Strasburg, 1960, Anderson et al., 1981). The
Apogonidae, which showed little discrimination in their richness between coral zones, include mainly macrophagic generalist species which migrate from reefs at night to feed (Hiatt and Strasburg, 1960; Hobson, 1974; Harmelin-Vivien, 1979).

In the absence of variation in the availability of shelter the hypothesis that decreasing live coral cover changes the nature of available prey is appealing. However, it fails to explain why the species richness of Scaridae and Acanthuridae (herbivores) increased significantly with higher live coral cover or why the planktivorous Pomacentridae, which were present at most sites, generally had higher abundances at the sites of maximum live coral cover. Concentrations of nitrates and phosphates in the lagoon were not correlated with the abundance of herbivores nor was the abundance of zooplankton correlated with the numbers of planktivores (Delesalle et al., in prep.). Hence the distribution of these fishes appears to reflect a dependence on live coral that is not yet quantified.

The most conservative interpretation of the multiple regression analyses shows that, while we cannot discount the effect of distance from the nearest ocean pass in organising fish communities in Mataiva Lagoon, live coral cover also has a significant effect. However, as distance does not remove any variance already accounted for by live coral cover the hypothesis that live coral cover, and not distance, is responsible for the patterns in our data is more parsimonious. Moreover, 


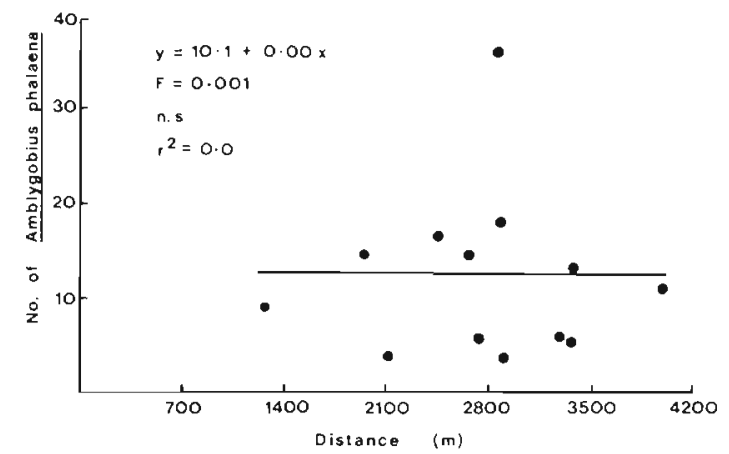

Fig. 6. Relationship between the abundance of Amblyogobius phalaena and distance from the nearest ocean pass or functional hoa

several features of the natural history of the situation support the idea that the main effects are not due to distance. The distances involved are small (Fig. 3) and unlikely to be a deterrent to mobile species like the Scaridae and Acanthuridae, and as there is a continuous network of reefs throughout the lagoon to provide shelter adult Chaetodontidae and Labridae should also be able to disperse within the lagoon. Furthermore, sedentary species which recruit from the plankton (e.g. Pomacentridae, Apogonidae and Gobiidae) are common to all sites indicating that larvae are well distributed throughout the lagoon. The abundance of the goby Amblygobius phalaena provides a test for the effect of distance from the nearest pass, in isolation from that of live coral cover. This species is assumed to have planktonic larvae ( $\mathrm{J}$. Leis, pers. comm.) and is unaffected by live or dead coral because it lives on the sand at the base of corals. If distance from a pass limits distribution of the larvae of $A$. phalaena we might expect its abundance to be negatively correlated with distance. However, linear regression showed no such relationship (Fig. 6)

Stochastic processes have been reasonably invoked to account for the structure of some coral reef fish communities (Sale, 1978; Talbot et al., 1978; Sale and Williams, 1982). Two pieces of evidence indicate that the amount of live coral cover, not chance, is responsible for the patterns of community structure we observed. Firstly, the slopes of regression lines for all 3 parameters are significantly different from zero (Fig. 4); secondly, the ordination shows that assemblages from replicates within a zone of live coral cover are more similar to one another than to those from different zones.

Our data indicate that the amount of live coral may be more important to fish than previously reported and infer that the complexity of coral-reef habitats should be considered in terms of a live coral and a structural component. A reduction in either component over relatively large areas of reef would therefore be expected to decrease the richness of the fish community. There are some distribution data to support this. Goldman and Talbot (1976) found 216 species associated with leeward (complex and rich in coral species) reef slopes and 89 at windward (stunted, species-poor) slopes. Similarly, Jones and Chase (1975) recorded 138 species from steep coral rich slopes and 91 from barrier reef flats and Harmelin-Vivien (1979) recorded 228 species from structurally complex, coral rich reef flats and 131 from dead coral rubble bank areas.

The influence of live coral on reef fish communities has implications for the management of these communities. Coral death (e.g. by sedimentation and crown of thorns infestations) is likely to cause a significant reduction in the number of fish species and individuals associated with a reef. These numbers should then decline further as the structure of the reef is eroded by physical forces.

Reese (1977) has argued that many of the Chaetondontidae have coevolved with corals and therefore the richness of this family makes a good indicator of the 'health' of coral reefs. Our data support his hypothesis. However, we submit that variation in species richness with increasing live coral cover is not restricted to the Chaetodontidae. Several families (e.g. Labridae and Gobiidae) showed this trend and the health of reefs may be equally well described by their richness or the richness of the whole community.

An important correlate of increasing live coral cover is increasing coral diversity in terms of both species and morphological types (Jokiel and Maragos, 1978). The effects of increases in coral richness and form on the structure of fish communities, as opposed to the effects of an increase in cover by a single coral species, obviously merit investigation.

Acknowledgements. We thank T. Lau and P. Hughes for help with analysis of data, and D. Hoese and J. Randall for aid in identifying taxa and checking nomenclature. B. Delesalle, D. Pollard, P. Sale, B. Salvat, H. Sweatman, M. Westoby and D. $\mathrm{McB}$. Williams provided critical discussions and comments on earlier drafts of the manuscript. The work was carried out with the help of G. I. E. Raro Moana (Contract Dam De No. 4500) and was supported by a French Government Scientific and Professional Scholarship and an Augmentative Research Support Grant from the Great Barrier Reef Marine Park Authority to J. D. B.

\section{LITERATURE CITED}

Anderson, G. R. V., Ehrlich, A. H., Ehrlich, P. R., Roughgarden, J. D., Russell, B. C., Talbot, F. H. (1981). The community structure of coral reef fishes. Am. Nat. 117: 476-495

Coles, S. L. (1980). Species diversity of decapods associated with living and dead reef coral Pocillopora meandrina. Mar. Ecol. Prog. Ser. 2: 281-291

Delesalle, B., Bagnis, R., Bell, J., Bennett, J., Denizot, M., 
Galzin, R., Montaggioni, L., Payri, C., Renon, J., Ricard, M., Vergonzanne, P. Etude de l'environnement lagonaire et récifal de l'atoll de Mataiva (Polynésie Française). (In prep.)

Frontier, S., Tbanez, F. (1974). Utilization d'une cotation d'abondance fondée sur une progression geométrique, pour l'analyse des composantes principales en ecologie planctonicue. J. exp. mar. Biol. Ecol. 14: 217-224

Gladfelter, W. B., Ogden, J. C., Gladfelter, E. H. (1980). Similarity and diversity among coral reef fish communities: a comparison between tropical western Atlantic (Virgin Islands) and tropical central Pacific (Marshall Islands) patch reefs. Ecology 61: 1156-1168

Goldman, B., Talbot, F. H. (1976). Aspects of the ecology of coral reef fishes. In: Jones, O. A., Endean, R. (ed.) Biology and geology of coral reefs. Vol. 4, Biology 2. Academic Press, New York, p. 125-154

Grovhoug, J. G., Henderson, R. S. (1978). Distribution of inshore fishes at Canton Atoll. Atoll Res. Bull. 221: 99-157

Harmelin-Vivien, M. L. (1979). Ichtyofaune des récifs coralliens de Tulear (Madagascar): ecologie et relations trophiques. These Doc. es - Sciences, Univ. Aix - Marseille

Hiatt, R. W., Strasburg, D. W. (1960). Ecological relationships of the fish fauna on coral reefs of the Marshall Islands. Ecol. Monogr. 30: 65-127

Hobson, E. S. (1974). Feeding relationships of teleostean fishes on coral reefs in Kona, Hawaii. Fish. Bull. U. S. 72: 915-1031

Jokiel, P. L., Maragos, J. E. (1978). Reef corals of Canton Atoll: II. Local distribution. Atoll Res. Bull. 221: 71-97

Jones, R. S., Chase, J. A. (1975). Community structure and distribution of fishes in an enclosed high island lagoon in Guam. Micronesica 11: 127-148

Luckhurst, B. E., Luckhurst, K. (1978). Analysis of the influence of substrate variables on coral fish communities. Mar. Biol. 49: 317-323

Randall, J. E. (1955). Fishes of the Gilbert Islands. Atoll Res. Bull. 47 : 1-243
Randall, J. E. (1967). Food habits of reef fishes of the WestIndies. Studies in Tropical Oceanography (Miami) 5: 665-847

Reese, E. S. (1977). Coevolution of corals and coral feeding fishes of the family Chaetodontidae. Proc. 3rd Int. Coral Reef Symp. 1: 267-274

Risk, M. J. (1972). Fish diversity on a coral reef in the Virgin Islands. Atoll Res. Bull. 153: 1-6

Sale, P. F. (1978). Coexistence of coral reef fishes - a lottery for living space. Environ. Biol. Fish. 3: 85-102

Sale, P. F., Dybdahl, R. (1975). Determinants of community structure for coral reef fishes in an experimental habitat. Ecology 56: 1343-1355

Sale, P. F., Dybdahl, R. (1978). Determinants of community structure for coral reef fishes in isolated coral heads at lagoonal and reef slope sites. Oecologia 34: 57-74

Sale, P. F., Williams, D. McB. (1982). Community structure of coral reef fishes. Are the patterns more than those expected by chance? Am. Nat. 120: 121-127

Talbot, F. H., Goldman, B. (1972). A preliminary report on the diversity and feeding relationships of the reef fishes on One Tree Island, Great Barrier Reef System. In: Proceedings of the Symposium on Corals and Coral Reefs, 1969 , Ernakulam, Cochin. Mar. Biol. Ass. India, p. 425-442

Talbot, F. H., Russell, B. C., Anderson, G. R. V. (1978). Coral reef fish communities: unstable high-diversity systems? Ecol. Monogr. 48: 425-440

Vivien, M. L. (1973). Contribution à la connaissance de l'ethologie alimentaire de l'ichtyofaune du platier interne des récifs coralliens de Tuléar (Madagascar). Téthys 5 (Suppl.): 221-308

Watling, L., Kinner, P. C., Maurer, D. (1978). The use of species abundance estimates in marine benthic studies. $J$. exp. mar. Biol. Ecol. 35: 109-118

Williams, W. T. (1976). Pattern analysis in agricultural science. Elsevier, New York 